\title{
On the Role of Affective Properties in Hedonic and Discriminant Haptic Systems
}

\author{
Matteo Bianchi1,2 (10) - Gaetano Valenza ${ }^{2}$ - Antonio Lanata ${ }^{2}$ - Alberto Greco ${ }^{2}$ • \\ Mimma Nardelli ${ }^{2}$ - Antonio Bicchi ${ }^{1,2}$ - Enzo Pasquale Scilingo ${ }^{2}$
}

Accepted: 1 July 2016 / Published online: 18 July 2016

(C) Springer Science+Business Media Dordrecht 2016

\begin{abstract}
Common haptic devices are designed to effectively provide kinaesthetic and/or cutaneous discriminative inputs to the users by modulating some physical parameters. However, in addition to this behavior, haptic stimuli were proven to convey also affective inputs to the brain. Nevertheless, such affective properties of touch are often disregarded in the design (and consequent validation) of haptic displays. In this paper we present some preliminary experimental evidences about how emotional feelings, intrinsically present while interacting with tactile displays, can be assessed. We propose a methodology based on a bidimensional model of elicited emotions evaluated by means of simple psychometric tests and statistical inference. Specifically, affective dimensions are expressed in terms of arousal and valence, which are quantified through two simple one-question psychometric tests, whereas statistical inference is based on rank-based
\end{abstract}

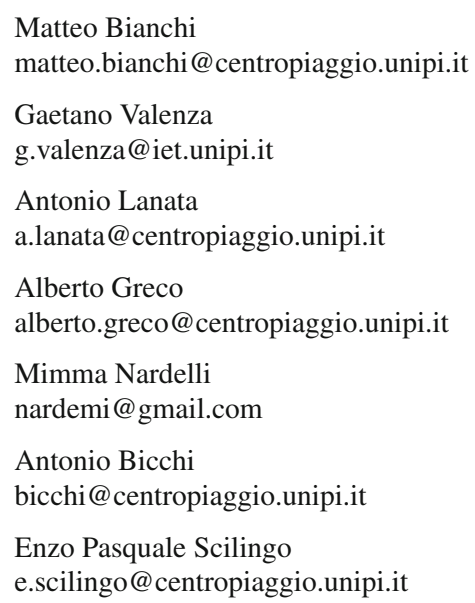

1 Department of Advanced Robotics, Istituto Italiano di Tecnologia, Via Morego 30, 16163 Genova, Italy

2 Research Centre "E. Piaggio”, University of Pisa, Largo Lucio Lazzarino 1, 56122 Pisa, Italy non-parametric tests. In this work we consider two types of haptic systems: (i) a softness display, FYD-2, which was designed to convey purely discriminative softness haptic stimuli and (ii) a system designed to convey affective caresslike stimuli (by regulating the velocity and the strength of the "caress") on the user forearm. Gender differences were also considered. In both devices, the affective component clearly depends on the stimuli and it is gender-related. Finally, we discuss how such outcomes might be profitably used to guide the design and the usage of haptic devices, in order to take into account also the emotional component, thus improving system performance.

Keywords Tactile displays · Affective haptics · Human experiments

\section{Introduction}

It is well-known that touch is the most developed sensory modality at birth and underpins cognitive developments throughout infancy and childhood [1]. Historically, researchers have viewed this sense as generally subserving a primarily discriminative role [2]. However, it is widely accepted that the multimodal properties of the human somatosensory system provide not only well-recognized discriminative inputs to the brain, but also an affective one [3]. Indeed, previous findings provide physiological and behavioral evidence that sensitivity to pleasant touch emerges early in development and, therefore, plays an important role in regulating human social interactions [4]. With regard to the communication of emotion, two general claims have been offered regarding touch. First, touch is thought to communicate the hedonic tone of emotion [5]. Second, touch is thought to be an intensifier of emotion-related communica- 
tion. Humans have a rapid first touch system, with obvious advantages for discriminative and sensory motor functions, and a slow second touch system, which represents the affective touch $[3,6-8]$.

Pleasantness perception seems to be strongly influenced by various haptic properties such as average level of surface roughness, average friction force occurring during fingertip stimulation, softness and smoothness, etc. $[9,10]$. Of note, these dependencies are affected by age, gender, body sites, velocities and forces of stimulus application [10,11]. At the same time, such a type of investigation has driven the development of haptic devices that are able to stimulate and influence user's emotional state, e.g. in [8,12-15]. In [8], authors presented a small, animal-like robot (the "Haptic Creature"), which was able to haptically perceive the external environment and to express its state through actions such as purring patterns and breathing rate. The effectiveness of the robot in communicating information on its emotional state was demonstrated in [14]. In [15], the authors provided a touch dictionary of gestures chosen by participants to convey emotions to a haptic system. In [16], a kinaesthetic haptic device was used by subjects to emotionally categorize visual stimuli, showing a role of the haptic system in modifying the user's emotional state. In [17] the authors presented the results of a pilot study that sought to assess the feasibility and effect of using a robotic companion animal called $\mathrm{CuDDler}$ on engagement and emotional states of five older adults with dementia living in nursing home care. Considering robot touch and tactile interaction between humans and robots, in [18] the authors demonstrated that both touches from the robot to the participants and touches from the participants to the robot facilitated their efforts. In [19] the authors implemented an algorithm to classify six emotions and six social messages transmitted by humans when touching a full-sized mannequin arm covered with touch-sensitive artificial skin. These experimental outcomes pioneered the description of particular affective touch systems, with final goal of devising guidelines for future design of social robots, human-robot interaction and communication, and emotional haptic devices. However, a standard, widely accepted procedure to characterize any haptic display per se under an emotional light still lacks, especially for haptic devices that are specifically developed to elicit discriminative haptic sensations exclusively. In other words, although physiology tells us that an affective component is always present in the sense of touch, such a component has rarely been taken into account for the device design, development and characterization. Of note, such a hedonic component might play a crucial role in influencing system performance in stimuli conveying. Therefore, in this study, our hypothesis is that an affective counterpart always exists in stimuli delivered through haptic devices, although designed to convey discriminative information exclusively (e.g. softness). In this work, we propose a characterization procedure of haptic-driven emotional states in humans as a function of the device physical parameters. To this aim, we describe how to adapt complex theories of emotions through three basic concepts: a two-dimensional model of emotions, two simple psychometric tests, and statistical inference. Specifically, we choose to express emotions through a simplified version of the so-called Circumplex Model of Affect (CMA) [20]. With this model, emotions can be represented as a simple combination of two dimensions conceptualized by the terms of valence and arousal. Valence is related to the pleasure/displeasure of the stimulus, whereas the arousal is related to the perceived strength of the stimulus. Of note, these dimensions can be quantified through a simple one-question psychometric test (see details below).

Here, we first extend the analysis described in [13], where an affective device able to administer "caress-like" (thus emotional) stimuli was considered, by including a larger number of users and taking into account gender differences. Then, we push forward this analysis by studying a tactile display $[21,22]$ which conveys softness stimuli by modulating the stretching state of a fabric. Importantly, while the first display [13] was specifically developed to elicit emotions through caress-like stimuli, the second display [21] was developed to provide discriminative sensations exclusively. In this study, we asked participants to assess six combinations of force and velocity stimuli associated to "strength of the caress" and "velocity of the caress", respectively, for the first display, and three stretching states of the fabric for the second display (i.e. stiffness levels) in terms of arousal and valence. Gender differences are also taken into account. Results show that, despite the kind of haptic sensation the systems elicit (discriminative or affective), both devices convey stimuli that are able to provide affective inputs to the users, well defined in terms of arousal and valence scores. In the last section of this paper, we discuss our experimental outcomes aiming to open novel endeavors for the future design of haptic systems in human-robot interaction.

\subsection{The Circumplex Model of Affect (CMA)}

In the literature, several theoretical models of emotions have been proposed [23]. Bringing concept from quantitative psychometrics, a commonly used mathematical/computational model of emotion is Russel's Circumplex Model of affect [24]. This model suggests a two affective dimensions, referring to the concepts of arousal and valence (Fig. 1). The valence dimension refers to the pleasantness of the stimulus, whereas the arousal dimension quantifies the corresponding physiological activation and expresses the intensity of the emotion. With this model, each emotion is described by a linear combination of arousal and valence levels (see Fig. 1). In order to quantify these affective dimensions, a graphic system, known as Self-Assessment Manikin (SAM) 


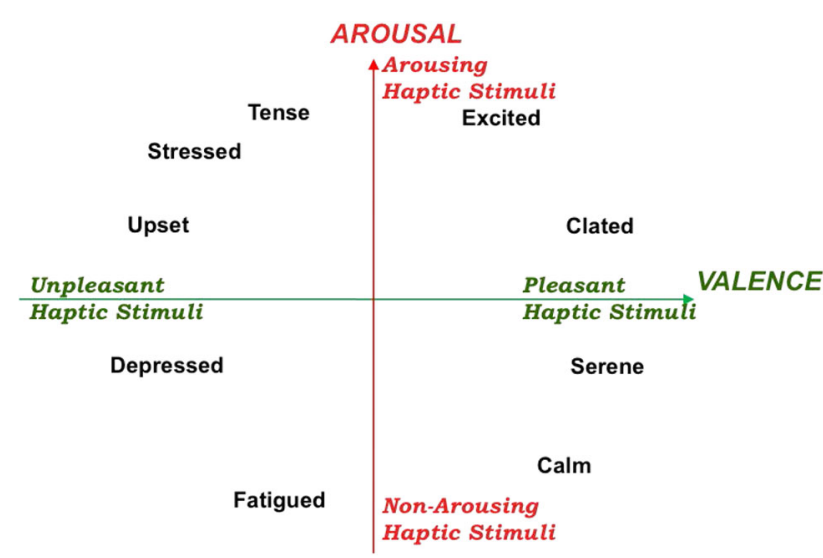

Fig. 1 The circumplex model of affect (CMA)

[25] was proposed. SAM goes from a smiling and happy figure to a sad one for indicating the valence of the emotion, while for the arousal dimension the SAM figures are excited for the major activation or relaxed for the opposite. Subjects are asked to choose between five levels of arousal (from not at all intense to very intense, from 0 to 4 , with 0 as neutral level) and between five levels of valence (from very unpleasant to very pleasant, from -2 to 2 , with 0 as neutral level). Of note, CMA modeling and SAM assessment has been widely used to understand physiological correlates of emotions and mental disorders [26-28]. Among the examples or applications of CMA in human-machine interaction, let us mention e.g. [29], where the authors presented a computational approach to generate and recognize affective hand movements, or [30], where the valence-arousal circumplex model description was used to investigate the capability of a non-humanoid robot to express artificial emotions in a meaningful manner to humans.

\section{Tactile Displays}

In this work, we consider two types of haptic devices: the first one is a softness display designed for discriminative touch [21]; the second one is a haptic system that reproduces a caress-like stimulus on the user forearm, which was thought and characterized in terms of affective haptics [13].

\subsection{Discriminative Touch: Fabric Yielding Display (FYD-2)}

The softness display, hereinafter referred to as FYD-2, is a fabric-based softness display that can reproduce object compliance controlling the stretching state of the fabric. More specifically, we connected the extremities of the elastic fabric to two rollers. Each roller can be moved in an independent fashion through a pulley placed on a motor shaft. The rotation

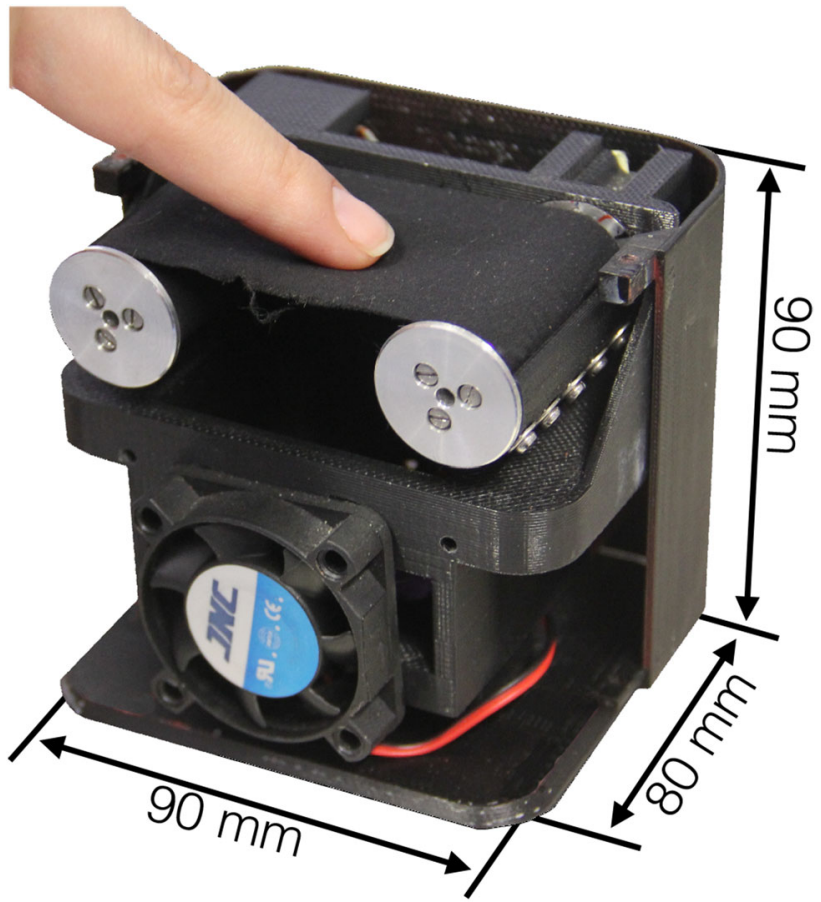

Fig. 2 A subject fingertip interacting with the FYD-2

of the motors produces a stretching or shrinking of the fabric, and thus increasing or decreasing its stiffness: when the two motors rotate in the same direction, they stretch the fabric, thus increasing its stiffness according to the motor angular position $\theta$. A view of the system is shown in Fig. 2.

The system is also able to on-line measure the contact area between the finger pad and the fabric, by exploiting a web-camera, which was placed just beneath the fabric, and a binarization algorithm, and the normal force exerted by the finger interacting with the fabric, through a load cell placed at the base of the device. In this manner, FYD-2 was proven to be able to reproduce arbitrary force-area curves of different specimens, according to the contact area spread rate (CASR) [31] paradigm, which states that a large part of cutaneous information used for softness discrimination is retained in the relationship between the indenting force and the contact area spread over the user finger pad.

FYD-2 was also characterized through experiments with humans and psychophysical methods, showing a good performance in eliciting a correct softness discrimination in users. For further information the reader is invited to refer to [21] and $[32,33]$.

\subsection{Affective Touch: A Device for "Caress-like" Haptic Stimuli}

The affective touch display [13] exploits the elasticity of a fabric to reproduce the haptic stimuli that are commonly conveyed through the human caress. More specifically, the user 


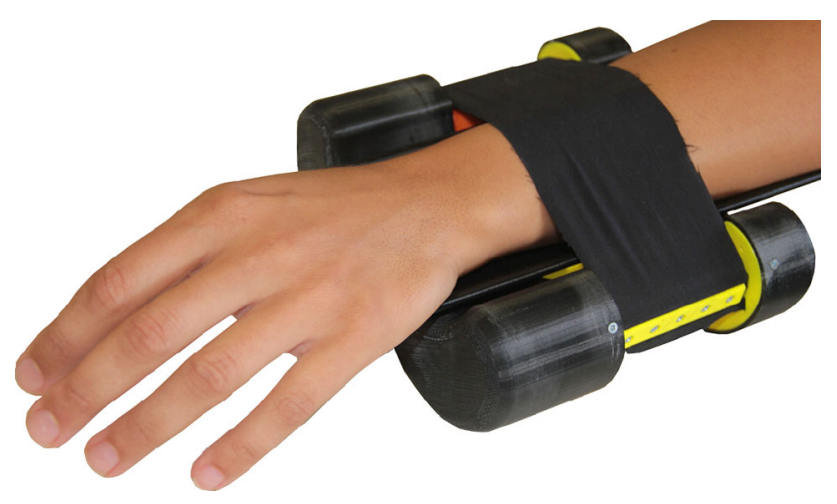

Fig. 3 The affective haptic system

places the forearm on the forearm support under the fabric layer, whose extremities are connected to two motors through two rollers. By controlling motor positions and rotation velocity, it is possible to change force and the velocity of the distributed haptic stimulation on the user's arm. A load cell allows to measure the normal force that the fabric exerts on the user's forearm. After a Calibration Phase, where the offset due to the forearm weight is removed, the exerted force (i.e. the strength of the caress) can be varied by acting on the two motor positions, which determine how much the fabric is wrapped around the forearm and hence the force exerted on it (maximum force $20 \mathrm{~N}$ ). When the target force level is reached (both motors are in the reference positions), we feed the motors using a sinusoidal trajectory as input and exploiting a built-in-position controller. Setting the frequency of the input we can control the velocity of motor rotation, i.e. the velocity of the caress, while the amplitude of the sinusoidal input determines the amplitude of motor rotation (maximum angular displacement of the motors is $\pm 90^{\circ}$ from the reference position). The duration of the entire control cycle is 1 ms.

An overview of the system is shown in Fig. 3. For further technical details on the device, the reader is invited to refer to [13].

\section{Experimental Setup}

Two experimental studies were carried out, one for each device. In Study 1, we considered FYD-2, while in Study 2 the Haptic Device for Caress-like stimuli. Each study was performed by a different group of participants. In Study 1, thirty-two right-handed participants aged $26 \pm 3(16 \mathrm{M})$ gave their informed consent to take part in the study. In Study 2, thirty-two right-handed participants aged $27 \pm 2(16 \mathrm{M})$ gave their informed consent to take part in the study. No participant of Study 1 and Study 2 reported any physical limitation, experience of chronic disease or personality/mental disorder that would have affected the experimental outcomes. These studies were approved by the Ethical Committee of the University of Pisa. Participants were informed about the protocol and about the purpose of the study, but they were not informed about the device physical parameter levels they would have underwent through.

\subsection{Study 1: FYD-2 Tactile Softness Display}

In this experiments, we asked participants, who were comfortably seated, to discriminate the softness of FYD-2 surface, using the index finger of their dominant hand as a probe. We considered three different positions $(\theta)$ of the motor corresponding to three different stiffness characteristics (i.e. $\theta=10^{\circ}$, corresponding to $0.27 \mathrm{~N} / \mathrm{mm}$, hereinafter referred to as LS; $\theta=50^{\circ}$, corresponding to $0.63 \mathrm{~N} / \mathrm{mm}$, hereinafter referred to as MS; $\theta=80^{\circ}$, corresponding to $0.9 \mathrm{~N} / \mathrm{mm}$, hereinafter referred to as HS). The inter-stimuli interval was $1 \mathrm{~s}$ while stimulus exploration lasted $10 \mathrm{~s}$. After each stimulus, participants were asked to assess the stimulus in terms of arousal and valence scores. The presentation of the stimuli were randomized among subjects.

\subsection{Study 2: Haptic Device for "Caress-like" Stimuli}

For all trials, participants were comfortably seated and wore earplugs. They were instructed to keep their right forearm horizontal and placed it on the forearm support, with hand palm down. The load cell was auto calibrated with respect to the forearm weight prior of each experimental trial. We used 6 different combinations of stimuli among 2 levels of force and 3 levels of velocity. The forces exerted by the fabric was one light of $2 \mathrm{~N}$ and one strong of $6 \mathrm{~N}$ (hereinafter referred to as LF and HF, respectively), the velocities of the caresses were 9.4, 37 and 65 (hereinafter referred to as, LV, MV and $\mathrm{HV}$, respectively), which correspond to three sinusoidal input trajectories, at the frequencies of $0.1,0.4$, and $0.7 \mathrm{~Hz}$, respectively. Between two stimuli the motors were stopped and the force was set to $0 \mathrm{~N}$ (fabric lightly contacts the forearm). To increase force, the motors wrap the fabric more closely around the forearm. In this case, the objective is to simulate a human caress performed with a non-negligible force, when the hand wraps the forearm and friction increases. This provokes skin torsion-no more pure sliding as for the light force condition. We selected the range of force in a preliminary informal piloting to avoid painful stimuli. At the beginning of the experimental protocol, a two-minutes resting state session was recorded. Then, all the six combinations of velocities and forces were administered to the participants, with a pre-stimulus and a post-stimulus interval of $35 \mathrm{~s}$ each. After each post-stimulus, the subjects were asked to assess the stimulus in terms of arousal and valence scores, within 
a time window of $20 \mathrm{~s}$. The presentation of the six kinds of caress-like stimuli were randomized among subjects.

\section{Experimental Results}

Experimental results are derived from the statistical analysis performed on the valence and arousal scores gathered from the healthy volunteers enrolled for the studies. In particular, non-parametric tests were employed since data resulted non-normally distributed ( $\mathrm{p}$ values gathered from Kolmogorov-Smirnov test with null hypothesis of normality resulted $<0.05$ ). Statistical analysis for FYD-2 in Study 1 deals with values of arousal and valence related to the three different levels of $\theta$. Accordingly, in this case we tested the null hypothesis of having an equal median between the three levels of $\theta$ through the Kruskal-Wallis non-parametric test. Results show significant differences between the participants' scores on both arousal and valence dimensions. Figure 4 shows that the higher is $\theta$ (i.e. the stiffer is the fabric), the higher is the arousal score given by subjects. Of note, an opposite trend was found on the valence dimension (i.e., higher $\theta$ values are associated to more unpleasant stimuli). A post-hoc test using Bonferroni correction was also carried out in order to investigate pairwise differences. Concerning the arousal and considering all the participants, comparison of low vs. high stiffness (i.e., LS vs. HS) and medium vs. high stiffness (i.e., MS vs. HS) resulted in statistically different scores with a p value $<0.001$. This trend was found


Fig. 4 FYD-2 stimuli. Boxplots of arousal (top first row, A) and valence (bottom second row, V) scores. Scores are averaged among $\theta$ values (LS, MS, HS) on the whole group (32 subjects, left panel), on the men group (16 subjects, central panel), and on the women group (16 subjects, right panel). p values: $* 0.01 ; * * * 0.005 ; * * * * 0.001$. p values after Kruskal-Wallis test are also reported for each group (significant $\mathrm{p}$ values in red). (Color figure online) in women while only the difference between MS and HS is significant $(p<0.01)$ in men. For the valence, statistical post-hoc analysis carried on all the subjects showed significant differences in scores between HS and the other levels MS, LS $(p<0.01)$. However, splitting the dataset in male and female subject groups, these pairwise differences vanish after the Bonferroni correction.

In Study 2, we analyzed arousal and valence scores to identify significant statistical differences between the two levels of force (LF and HF) and the three velocity levels (LV, MV, HV) of the caress-like stimuli. Accordingly, we tested the null hypothesis of having an equal median between the two levels of force through the Mann-Whitney non-parametric test, whereas for the three levels of velocity we used the Kruskal-Wallis non-parametric test. Concerning the force of the caress, we found that the lowest level of $2 \mathrm{~N}$ corresponded to the lowest arousal but the highest (pleasant) valence perception, but they were not confirmed looking at differences in gender, see Fig. 5). Concerning the velocity of the caress, higher velocities were associated to higher arousal and lower (unpleasant) valence scores (Fig. 6).

A post-hoc analysis with Bonferroni adjustment was carried out for the velocities. Results on the arousal scores gathered from the whole group revealed a significant difference between the velocity levels LV and HV exclusively, with $p<0.01$. Of note, the analysis on data from female participants showed similar results (with $p<0.01$ ), whereas no significant differences were found in the male group. On the valence dimension, both the comparisons between $\mathrm{LV}$ versus $\mathrm{MV}$ and $\mathrm{HV}$ velocities were significantly different, with a $\mathrm{p}-$ value $<10^{-5}$. Looking at the gender differences, the same significant differences were found for both male and female subject groups but with higher $\mathrm{p}$-values for the female group $(p<0.01)$.

Of note, we also evaluated the interaction effects between velocity and force on both arousal and valence dimensions, but they resulted not statistically significant $(p>0.05)$.

\section{Discussions}

Results from Study 1 showed that there is a congruent trend in the arousal scores with the stiffness levels, i.e. the higher the stiffness, the higher the arousal, and an opposite trend for the valence, i.e. the higher the stiffness the lower the valence score. This means that a stiffer stimulus can be associated to a more intense and unpleasant emotional feeling, which is in line with what is reported in the literature, see e.g. [10]. Gender differences were observed in arousal scores. Looking at the post-analysis results, it is worthwhile noting that for both male and female participants, the arousal scores associated to the high stiffness level (HS) w.r.t, the medium (MS) stiffness level is statistically significant. This means that HS can be 



Fig. 5 Caress-like haptic system. Boxplots of arousal (top first row, A) and valence (bottom second row, $\mathbf{V}$ ) scores. Scores are averaged among force levels (LF and HF) on the whole group (32 subjects, left panel), on the men group (16 subjects, central panel), and on the women group (16 subjects, right panel). $\mathrm{p}$ values after Kruskal-Wallis test are also reported for each group significant (significant $\mathrm{p}$ values in red). (Color figure online)
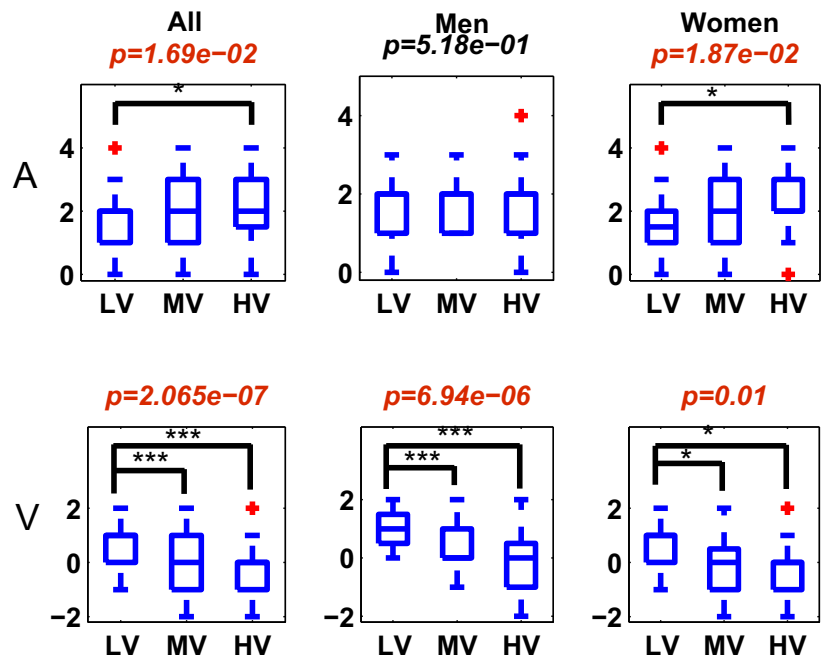

Fig. 6 Caress-like haptic system. Boxplots of arousal (top first row, A) and valence (bottom second row, $\mathbf{V}$ ) scores. Scores are averaged among velocity levels ( $\mathrm{LV}, \mathrm{MV}, \mathrm{HV}$ ) on the whole group (32 subjects, left panel), on the men group (16 subjects, central panel), and on the women group (16 subjects, right panel). p values: $* 0.01 ; * * 0.005 ; * * * 0.001$. p values after Kruskal-Wallis test are also reported for each group (significant p-values in red). (Color figure online)

discriminated by participants in terms of emotional intensity w.r.t (no further gender difference was observed). Of note, LS was perceived significantly different from HS by women only.

Significant differences observed for the whole group for the arousal scores can also be noticed under the valence point of view. Therefore, these levels can elicit significant differ- ent pleasantness emotional feelings in users, even if gender differences are present. The fact that gender differences are present at the valence level can be found also in the literature [10]. From Study 1 the following conclusions can be drawn:

1 During the interaction with the tactile display the emotional feeling strictly depends on the stiffness workspace. This perceptual cue can help to guide the design of the system, e.g. to avoid that most unpleasant stimuli are provided for long periods. This fact can play a relevant role in applications with wearable systems, e.g. to increase acceptability. In other terms, it would be important to individuate the suitable emotional workspace of the devices related to the particular task. For example, to convey alarm signals through tactile stimulation, unpleasant high-intensity emotional level stimuli might be recommended. At the same, it would be interesting to characterize the systems in terms of emotional Just Noticeable Difference (JND), i.e. the minimum amount of stimulus capable of eliciting an emotional feeling in users;

2 Gender differences in the target users should be taken into account in the design of haptic systems, i.e. the emotional JND might be gender-related.

Similar conclusions can be also drawn from Study 2, even if in this case the device was specifically thought to elicit affective stimulation. In addition to tactile perception, stimuli in this case are also intended to convey an emotional content: looking at gender differences, if we consider the strength of the caress, we found differences only in arousal scores for men and only in valence scores for women, respectively. In other terms, male participants felt a more emotionally intense stimulus as the strength of the caress increased, while female participants felt stronger caresses as more unpleasant, which is coherent with [34].

Concerning the velocity of the caress we found that the slower the caress the more pleasant is the stimulus for both men and women, which is coherent with [35], while for women the faster the caress the more emotional intense is the stimulus. Furthermore, the decrease of the scores of valence with the increasing force is coherent with the findings observed in several previous studies, such as in [36], where a linear relationship was found between the velocity and the intensity of the caress that can be explained by the activity of myelinated fast conducting fibers (A beta).

Post-hoc analysis results show that the difference between $\mathrm{LV}$ and HV arousal scores was noticed in females but not in males. On the valence dimension, differences bw. LV and MV and LV and HV for all the groups. Results suggest a possible different emotional JND for men and women in terms of intensity that further sustain the role of participant gender in the elicited emotions, which should be taken into account. 
The gender differences we observed in arousal scores for both studies confirm previous evidences in the literature reporting different responses by the two sexes to arousing stimulation. Taylor et al. suggested a physiological response linked to a pattern of "tend-and-befriend" in females, while a "fight-or-flight" pattern related to sympathetic arousal occurs in the males [37]. Such gender differences could be related to different neural correlates reported in previous works $[38,39]$. Neuroimaging studies, in fact, demonstrated sexrelated differences in autonomic cardiac control through activity of brain regions such as amygdala, the anterior insula, the anterior cingulate cortex and the orbitofrontal cortex. Importantly, neural activity of these brain regions significantly affect measures, defined in time and frequency domains, extracted from HRV series [40,41]. Moreover, while a positive correlation between the regional cerebral blood flow in amygdala and parasympathetic indices from HRV series was found in females, a negative correlation was found in males [41].

\section{Conclusions and Future Works}

In this paper, we have presented a general framework to evaluate the emotional counterpart of haptic stimuli conveyed by haptic systems. Such a procedure is based on CMA and exploits simple statistical tools. We have applied such a technique to two haptic systems, one properly designed to enable discriminative haptic stimuli recognition (in terms of softness), the second one to convey caress-like affective stimuli. Results show that, despite the type of haptic stimuli to be conveyed, both devices elicit emotional response correlated to stimulus parameters.

The importance to correctly assess the emotional response in devices thought for human-robot interaction is important and can have a strong impact in this field. For example, for long duration interaction (e.g. assistive devices for prosthesis users or visually impaired people), the rendering of stimuli that are perceived with a good level of valence could be chosen as a successful strategy to enhance user acceptability. At the same time, devices that are able to render high arousal stimuli could be successfully employed to convey non-verbal alarm signals. All these aspects will be investigated in future works: the goal of this paper is to lay the foundations of a novel paradigm to assess - and consequently exploit - the affective component in haptic interaction between human and artificial side.

Considering haptic systems suitably designed to convey affective stimuli, the correct classification of the emotional response they are able to elicit could represent a crucial step, e.g. to tailor specific neuro-rehabilitation therapies (based on haptic stimulation) or to perform effective stimulation in naturalistic environment. Finally, what is important to notice is that gender differences play an important role on the emotional response associated to haptic stimuli: the procedure described in this paper could be hence useful in devising guidelines to drive the design of gender-specific haptic systems, to increase the effectiveness in stimulus rendering as well as acceptance. This point will be investigated as future work.

In conclusion, the here reported results can pave the path towards a novel paradigm of human robot interaction in social robotics. By definition, social robots should be able to interact with people in a natural and interpersonal manner often to achieve social-emotional goals [42]. To fully accomplish this objective, social robots need to communicate naturally with people using both verbal and nonverbal signals, engaging human users also on an emotional level. Providing simple techniques to characterize the emotional aspects related to (non) verbal communication, as touch-mediated stimulation actually is, can open novel insights for a successful cooperation between humans and robots. Indeed, understanding the emotional counterpart of such a kind of communication can offer useful guidelines and decisional criteria to design and choose the most effective stimulation able to elicit suitable emotions in users, taking into account emotion intensity, pleasantness and, ultimately, user's acceptability. In this manner, we can do a significant step further towards a novel generation of robots that can successfully play a beneficial role in the daily lives of people, in a wide range of application fields, including but not limiting to assistive robotics, collaboration and entertainment, among the others.

Acknowledgements This work is supported in part by the European Research Council under the Advanced Grant "SoftHands: A Theory of Soft Synergies for a New Generation of Artificial Hands" (No. ERC291166) and by the EU FP7 project (No. 601165) "WEARable HAPtics for Humans and Robots (WEARHAP)".

\section{References}

1. Hertenstein MJ (2002) Touch: its communicative functions in infancy. Hum Dev 45(2):70-94

2. Mountcastle VB (2005) The sensory hand: neural mechanisms of somatic sensation. Harvard University Press, Cambridge

3. McGlone F, Wessberg J, Olausson H (2014) Discriminative and affective touch: sensing and feeling. Neuron 82(4):737-755

4. Fairhurst MT, Löken L, Grossmann T (2014) Physiological and behavioral responses reveal 9-month-old infants sensitivity to pleasant touch. Psychological Sci 25(5):1124-1131

5. Hertenstein MJ, Keltner D, App B, Bulleit BA, Jaskolka AR (2006) Touch communicates distinct emotions. Emotion 6(3):528

6. Stiehl WD, Lieberman J, Breazeal C, Basel L, Lalla L, and Wolf M (2005) "Design of a therapeutic robotic companion for relational, affective touch. In: Robot and Human Interactive Communication, 2005. ROMAN 2005. IEEE international workshop on. IEEE, pp. 408-415

7. Rolls ET (2010) The affective and cognitive processing of touch, oral texture, and temperature in the brain. Neurosci Biobehav Rev 34(2):237-245 
8. Yohanan S, Hall J, MacLean K, Croft E, der Loos MBMV, Chang J, Nielsen D, Zoghbi S (2009) Affect-driven emotional expression with the haptic creature. In Proceedings of UIST, User Interface Software and Technology

9. Klöcker A, Oddo CM, Camboni D, Penta M, Thonnard J-L (2014) Physical factors influencing pleasant touch during passive fingertip stimulation. PloS One 9(7):e101361

10. Essick GK, McGlone F, Dancer C, Fabricant D, Ragin Y (2010) Quantitative assessment of pleasant touch. Neurosci Biobehav Rev 34(2): 192-203

11. May AC, Stewart JL, Tapert SF, Paulus MP (2014) The effect of age on neural processing of pleasant soft touch stimuli. Front Behav Neurosci 8:52

12. Tsetserukou D (2010) Haptihug: a novel haptic display for communication of hug over a distance. Eurohaptics Conf 2010:340-347

13. Bianchi M, Valenza G, Serio A, Lanata A, Greco A, Nardelli M, Scilingo E, Bicchi A (2014) Design and preliminary affective characterization of a novel fabric-based tactile display. In: Haptics Symposium (HAPTICS), 2014 IEEE, pp 591-596

14. Yohanan S, MacLean K (2011) Design and assessment of the haptic creature's affect display. In: In HRI '11 Proceedings of the 6th international conference on Human-robot interaction, pp 473-480

15. Yohanan S, MacLean KE (2012) The role of affective touch in human-robot interaction: human intent and expectations in touching the haptic creature. Int J Social Robot 4(2):163-180

16. Gatti E, Caruso G, Bordegoni M, Spence C (2013) Can the feel of the haptic interaction modify a user's emotional state? In Proceedings of World Haptics, pp 247-252

17. Moyle W, Jones C, Sung B, Bramble M, O’Dwyer S, Blumenstein M, Estivill-Castro V (2015) What effect does an animal robot called cuddler have on the engagement and emotional response of older people with dementia? a pilot feasibility study. Int J Soc Robot 8(1):145-156

18. Shiomi M, Nakagawa K, Shinozawa K, Matsumura R, Ishiguro H, Hagita N (2016) Does a robot's touch encourage human effort? Int J Soc Robot, pp 1-11

19. Silvera-Tawil D, Rye D, Velonaki M (2014) Interpretation of social touch on an artificial arm covered with an eit-based sensitive skin. Int J Soc Robot 6(4):489-505

20. Posner J, Russell J, Peterson B (2005) The circumplex model of affect: an integrative approach to affective neuroscience, cognitive development, and psychopathology. Dev Psychopathol 17(03):715-734

21. Bianchi M, Serio A (2015) Design and characterization of a fabricbased softness display. IEEE Trans Haptics 8(2):152-163

22. Serio A, Bianchi M, Bicchi A (2013) A device for mimicking the contact force/contact area relationship of different materials with applications to softness rendering. In Intelligent robots and systems (IROS), 2013 IEEE/RSJ international conference on Nov 2013, pp. 4484-4490

23. Calvo RA, D’Mello S, Gratch J, Kappas A (2014) The oxford handbook of affective computing. Oxford University Press, Oxford

24. Russell JA (1980) A circumplex model of affect. J Personal Soc Psychol 39(6):1161

25. Bradley MM, Lang PJ (1994) Measuring emotion: the selfassessment manikin and the semantic differential. J Behav Ther Exp Psychiatry 25(1):49-59

26. Valenza G, Citi L, Gentili C, Lanata A, Scilingo EP, Barbieri R (2015) Characterization of depressive states in bipolar patients using wearable textile technology and instantaneous heart rate variability assessment. IEEE J Biomed Health Inform 19(1):263-274

27. Valenza G, Lanat A (2012) Oscillation of heart rate and respiration synchronize during affective visual elicitation. IEEE Trans Inform Technol Biomed 16:683-690
28. Lanata A, Valenza G, Scilingo EP (2013) Eye gaze patterns in emotional pictures. J Ambient Intell Humaniz Comput 4(6):705715 Springer

29. Samadani A-A, Kubica E, Gorbet R, Kulić D (2013) Perception and generation of affective hand movements. Int J Soc Robot 5(1):3551

30. Novikova J, Watts L (2015) Towards artificial emotions to assist social coordination in hri. Int J Soc Robot 7(1):77-88

31. Bicchi A, De Rossi DE, Scilingo EP (2000) The role of the contact area spread rate in haptic discrimination of softness. IEEE Trans Robot Autom 16(5):496-504

32. Bianchi M, Scilingo EP, Serio A, Bicchi A (2009) A new softness display based on bi-elastic fabric. In: World Haptics Conference, pp 382-383

33. Bianchi M, Serio A, Scilingo EP, Bicchi A (2010) A new fabricbased softness display. In Proceedings IEEE Haptics symposium, pp 105-112

34. Löken LS, Wessberg J, McGlone F, Olausson H (2009) Coding of pleasant touch by unmyelinated afferents in humans. Nat Neurosci 12(5):547-548

35. Essick GK, McGlone F, Dancer C, Fabricant D, Ragin Y, Phillips N, Jones T, Guest S (2010) Quantitative assessment of pleasant touch. Neurosci Biobehav Rev 34(2):192-203

36. Löken LS, Evert M, Wessberg J (2011) Pleasantness of touch in human glabrous and hairy skin: order effects on affective ratings. Brain Res 1417:9-15

37. Taylor SE, Klein LC, Lewis BP, Gruenewald TL, Gurung RA, Updegraff JA (2000) Biobehavioral responses to stress in females: tend-and-befriend, not fight-or-flight. Psychol Rev 107(3):411

38. Huikuri HV, Pikkuja SM, Airaksinen KJ, Ika MJ, Rantala AO, Kauma H, Lilja M, Kesa YA et al (1996) Sex-related differences in autonomic modulation of heart rate in middle-aged subjects. Circulation 94(2):122-125

39. Kuo TB, Lin T, Yang CC, Li C-L, Chen C-F, Chou P (1999) Effect of aging on gender differences in neural control of heart rate. Am J Physiol Heart Circ Physiol 277(6):H2233-H2239

40. Wong SW, Kimmerly DS, Massé N, Menon RS, Cechetto DF, Shoemaker JK (2007) Sex differences in forebrain and cardiovagal responses at the onset of isometric handgrip exercise: a retrospective fmri study. J Appl Physiol 103(4):1402-1411

41. Nugent AC, Bain EE, Thayer JF, Sollers JJ, Drevets WC (2011) Sex differences in the neural correlates of autonomic arousal: a pilot pet study. Int J Psychophysiol 80(3):182-191

42. Breazeal C, Takanishi A, Kobayashi T (2008) Social robots that interact with people. In: Siciliano B, Khatib O (eds) Springer handbook of robotics. Springer, Berlin, pp 1349-1369

Matteo Bianchi Ph.D. is currently research fellow at Istituto Italiano di Tecnologia/ADVR and University of Pisa - Research Center "E. Piaggio", and Research Affiliate at Mayo Clinic (Rochester, USA). His research interests include haptic interface design, with applications in medical robotics and assistive/affective human-robot interaction; human and robotic hands; optimal sensing; human-inspired control for soft robots; psychophysics and mathematical modelling of the sense of touch and human manipulation. He is author of contributions to international conferences, journals and books and recipient of several national and international awards in these fields. He serves as reviewer and member of the editorial board and organizing committee of international journals and conferences. 
Gaetano Valenza Ph.D. is currently research fellow at University of Pisa and Harvard Medical School/ Massachusetts General Hospital, Boston, USA. The main topics of his research are statistical and nonlinear biomedical signal and image processing, cardiovascular and neural modeling, and wearable systems for physiological monitoring. Applicative fields include the assessment of autonomic nervous system activity on cardiovascular control, brain-heart interactions, affective computing, assessment of mood and mental disorders, and disorder of consciousness. He is author of more than 100 international scientific contributions in these fields published in peer-reviewed international journals, conference proceedings, and book chapters, and is official reviewer of more than forty international scientific journals. He has been involved in several international research projects, and currently is the scientific co-coordinator of the European project H2020-PHC-2015689691-NEVERMIND. He has been guest editor of several scientific journals, and is currently member of the editorial board of the Natures journal "Scientific Reports".

Antonio Lanata Ph.D. received the graduate degree in electronic engineering in 2001 and the Ph.D. degree in automation, robotics and bioengineering in the 2006 from University of Pisa, Pisa, Italy. Currently, he is with the Department of Information Engineering and with the Research Centre "E. Piaggio", Faculty of Engineering, at the University of Pisa. His research interests are focused on designing and developing wearable systems for biomedical applications and high-level signals processing. Main fields of application are affective computing, pervasive computing, mental and consciousness disorders, human interaction and eye information tracking. He is guest editor of the some international journals such as IEEE Journal of Biomedical Health Informatics and Frontiers in Neuroengineering. He has published numerous articles in international scientific journals and an author of several chapters of books.

Alberto Greco Ph.D. graduated in Biomedical Engineering from the University of Pisa (Italy), in 2010. He received a PhD degree in Automatics, Robotics and Bioengineering from University of Pisa in 2015. $\mathrm{He}$ is currently research fellow at Research Center "E. Piaggio", University of Pisa and he was a visiting fellow at the University of Essex, UK, in 2014. His main research interests are physiological modelling, wearable monitoring system, and biomedical signal processing. Applications include affective computing and the assessment of mood and consciousness disorders.

Mimma Nardelli received her Bachelor and Master degree in Biomedical Engineering at the University of Pisa in 2009 and 2012, respectively. Currently, she is a Ph.D student at the University of Pisa and works with the Research Center "E. Piaggio". Her research interests are mainly related to the study of nonlinear dynamics in physiological signals gathered from the central and autonomic nervous systems. Fields of application include affective computing, assessment of mood disorders and disorders of consciousness.
Antonio Bicchi Ph.D. is Senior Researcher at Istituto Italiano di Tecnologia/ADVR. He is also Professor of Robotics at the University of Pisa, and Adjunct Professor at Arizona State University. He graduated from the University of Bologna in 1988 and was a postdoc scholar at M.I.T. Artificial Intelligence lab between 1988 and 1991.He served as Director of the "Enrico Piaggio" Center for Bioengineering and Robotics of the University of Pisa between 2003 and 2012. His main research interestsare in Robotics, Haptics, and Control Systems. He has published more than 400 papers on international journals, books, and refereed conferences. He serves as Vice President for Publications in IEEE Robotics and Automation Society (RAS), has been appointed as founding Editor in Chief of the IEEE Robotics and Automation Letters, and will serve as Program Chair for ICRA 2016. He has been President of the Italian association of researchers in automatic control, Editor in Chief of the Conference Editorial Board for the IEEE RAS, as VP Membership and Distinguished Lecturer of IEEE RAS. He is Editor-in-Chief for the series "Springer Briefs on Control, Automation and Robotics," and has served in the editorial board of all top-ranked journals in Robotics (Int.1 J. Robotics Research, the IEEE Trans. on Robotics and Automation, IEEE Trans. Automation Science and Engineering, and IEEE RAS Magazine). He has organized and co-chaired the first World Haptics Conference (2005), and Hybrid Systems: Computation and Control (2007). He is the recipient of several awards and honors. In 2012, he was awarded with an Advanced Grant from the European Research Council for his research on human and robot hands. Antonio Bicchi is a Fellow of IEEE since 2005.

Enzo Pasquale Scilingo Ph.D. is an Associate Professor in Electronic and Information Bioengineering at the University of Pisa. He received the Laurea Degree in Electronic Engineering from the University of Pisa, Italy and the Ph.D.degree in Bioengineering from the University of Milan, in 1995 and 1998 respectively. Currently, he is pursuing his research work mainly at the Research Center "E. Piaggio" as well as at the Information Engineering Department of the University of Pisa. He coordinated a European project EC-FP7-ICT-247777 "PSYCHEPersonalised monitoring SYstems for Care in mental Health", and he is currently coordinating the European project H2020-PHC-2015- 689691 NEVERMIND - NEurobehavioural predictiVE and peRsonalised Modelling of depressIve symptoms duriNg primary somatic Diseases with ICT-enabled self-management procedures. His main research interests are in wearable monitoring systems, human-computer interfaces, biomedical and biomechanical signal processing, modelling, control and instrumentation. He is author of more than 150 papers on peerreview journals, contributions to international conferences and chapters in international books. He is currently serving as reviewer to many international journals and as member of Program and Scientific Committees of yearly international conferences. He is guest associate editor of Frontiers in Neuroengineering journal, guest editor of the Journal of Biomedical and Health Informatics, special issue on Sensor Informatics for Managing Mental Health, 2015, and associate editor of ETRI Journal. 